\title{
DC Field Response of One-Dimensional Flames Using an Ionized Layer Model
}

\author{
Yuan Xiong, Dae Geun Park, Bok Jik Lee, Suk Ho Chung, Min Suk Cha \\ Clean Combustion Research Center, King Abdullah University of Science and Technology, \\ Thuwal 23955-6900, Saudi Arabia
}

Email: min.cha@kaust.edu.sa

\begin{abstract}
We develop a simplified model to better explain electric current response when direct current (DC) is applied to a flame. In particular, different current responses have been observed by changing the polarity of the DC in a sub-saturated current regime that results from the presence of ions and electrons in the flame zone. A flame zone was modeled as a thin, ionized layer located in one-dimensional DC electric fields. We derived simplified model-governing equations from species equations by implementing mobility differences dependent on the type of charged particle, particularly between ions and electrons; we performed experiments to substantiate the model. Results showed that the sub-saturated current and local field intensity were significantly influenced by the polarity of the DC because of the combined effect of unequal mobility of charged particles and the position of the ionized layer in the gap relative to two electrodes. When an energized electrode is close to the ionized layer, applying a negative DC causes a more rapid increase in current than by applying a positive DC to the same electrode. Results from our experimental measurement of current using counterflow diffusion flames agreed qualitatively well with the model predictions. A sensitivity analysis using dimensional and non-dimensional parameters also supported the importance of the mobility difference and the relative location of the ionized layer on the electric current response.
\end{abstract}

Keywords: ion current, electrically assisted combustion, direct current, flame. 


\section{Introduction}

For decades, researchers have been investigating how electrically assisted combustion can be used to control the properties and behavior of both premixed and non-premixed flames. Charged particles of positive and negative ions and electrons are generated in a flame zone through chemi-ionization and subsequent ion chemistry. External electric fields can influence the movement of charged particles, causing adjustments to various aspects of combustion including flame stability [1-6], flame propagation speed [7-11] and emissions [12-16].

For example, under external electric fields charged particles gain their momentum due to Lorentz force. Collisional momentum transfer from the charged particles to neutrals leads to a modification of a local flow field, known as an ionic wind effect [1, 3, 17-20]. Such an ionic wind can be associated with an electric current due to external electric fields.

Typically, when an external DC field is applied, an electric current from a flame increases with the DC field [21,22]; it becomes saturated with an excessive DC field exceeding a certain critical field intensity [23-26]. In the sub-saturated regime, space charges redistribute to

minimize their potential, shielding the electric field and limiting the current, but in the saturated regime, no further space charges remain in the flame zone-all generated ions and electrons migrate toward their corresponding electrode. In this case, the saturated current is limited by the effective rate of charged particle generation in the flame [20,23]. Therefore, a saturated current is understood to be an intrinsic property of a flame with a given mixture, temperature, pressure and flow conditions.

However, in the sub-saturated regime, because the current response due to the separation of charged particles from a flame is a result of local field intensity, we cannot obtain a generalized ion current trend with respect to an external electric field. For example, when the polarity of a DC field to a flame is changed, one might observe a drastic change in the current, even with the same external voltage [10, 26-29]. Such a contrasting response of the ion current with respect to the polarity of the DC is sometimes referred to as a flame diode effect [10]. However, due to the lack of rigorous approaches to explain the behavior of the current in the sub-saturated regime, many observations regarding this regime and consequent differences in flame response remain unclear.

For this reason, we developed a simplified model and a complementary experiment to explore the characteristics of electric current from a thin, ionized layer with applied DC fields. We focus on how current behavior differs with different polarities in the sub-saturated regime. 


\section{A simplified model and simulation}

Our simplified model to predict the electric current considered an ionization layer in onedimensional space, with a coordinate $x$ domain of $[0, L]$, where $L$ is the distance between two parallel electrodes (Fig. 1). It is a transient diffusion system that generates charged particles. In modeling the drift-diffusion transport of charged multi-species with the ionization source under external electric fields, three groups of charged particles became evident: positive ions, negative ions and electrons.

Governing equations were derived based on a simplified model from a previous work [20], where only ions were included in the analysis of current behavior with flames. Here, we extended these governing equations to accommodate for the large difference in the mobility between ions and electrons because electrons account for a considerable portion of the negatively charged particles in flames. A convective effect on the movement of charged species was not modeled, because a typical ion drift velocity $(O(10 \mathrm{~m} / \mathrm{s})$ at $1 \mathrm{kV} / \mathrm{cm})$ is two orders of magnitude higher than a typical flow velocity in laminar flames $(O(0.1 \mathrm{~m} / \mathrm{s}))$. The

momentum transfer from charged species to background neutrals was also neglected. Although a modeling of the interaction between ions and neutrals is essential to observe an ionic wind [30], in the present one-dimensional model, accounting for ionic wind effects was not feasible. Nevertheless, the interaction might have minimal impact on electric current as long as the interaction does not result in quenching charges, which is not likely to happen with collisions between charged species and neutral molecules.

Charged particles were generated through chemi-ionization in a flame and modeled with a spatial Gaussian profile for the source terms on the basis of a local isothermal assumption of the reaction zone. Poisson's equation for electrostatic potentials was coupled with the charge transport equations and the resulting governing equations are as follows (see supplementary material for details):

$$
\begin{aligned}
& \text { Positive ions: } \frac{\partial n_{+}}{\partial t}+\frac{\partial}{\partial x}\left[-D_{+} \frac{\partial n_{+}}{\partial x}-K_{+} n_{+} \frac{\partial V}{\partial x}\right]=k_{i} g-k_{r} n_{+}\left(n_{e}+n_{-}\right), \\
& \text {Negative ions: } \frac{\partial n_{-}}{\partial t}+\frac{\partial}{\partial x}\left[-D_{-} \frac{\partial n_{-}}{\partial x}+K_{-} n_{-} \frac{\partial V}{\partial x}\right]=(1-\alpha) k_{i} g-k_{r} n_{+} n_{-},
\end{aligned}
$$


Electrons: $\frac{\partial n_{e}}{\partial t}+\frac{\partial}{\partial x}\left[-D_{e} \frac{\partial n_{e}}{\partial x}+K_{e} n_{e} \frac{\partial V}{\partial x}\right]=\alpha k_{i} g-k_{r} n_{+} n_{e}$

Electric potential: $\frac{\partial^{2} V}{\partial x^{2}}=-\frac{q_{e}}{\varepsilon}\left(n_{+}-n_{-}-n_{e}\right)$ and

Spatial profile of ionization: $g=\exp \left[-\frac{\pi(x-H)^{2}}{a^{2}}\right]$,

where $t$ is the time; subscripts,+- and $e$ represent the positive and negative ions and electrons, respectively; $n$ is the number density of charged species carrying a unit charge [31,32]; $K$ is the mobility; $D$ is the diffusion coefficient; $k_{i}$ is an ionization rate coefficient; $k_{r}$ is the recombination rate coefficient; $V$ is the electric potential; $H$ is the center of the ionized layer; $a$ is a thickness parameter in the Gaussian profile of the ionized layer; $q_{e}$ is the unit charge $\left(1.6 \times 10^{-19} \mathrm{C}\right)$; $\varepsilon$ is the vacuum permittivity $\left(8.854 \times 10^{-12} \mathrm{C} / \mathrm{V}-\mathrm{m}\right)$; and $\alpha$ is the ratio of electrons among the negatively charged species ranging between 0 and 1 . The mobility of charged species and the kinetic rates for ionization and recombination are described in Section 4.1.

The boundary conditions at $x=0$ and $L$ are

$$
V(0, \mathrm{t})=V_{a}, V(L, t)=0, n_{k}(0, t)=0, n_{k}(L, t)=0,
$$

where $V_{a}$ is the electric potential applied to the electrode located at $x=0$.

This system of equations was solved with the ordinary differential equation solver ODE23t in MATLAB software using the method of lines approach. A first-order upwind discretization was used on clustered grid points near the boundaries and the location of the ion source [20]. Grid convergence, based on Richardson extrapolation, was verified with three consecutively refined grid points from 100 to 400, and 200 grid points was selected.

\section{Experiment}

We conducted a counterflow burner experiment to substantiate the results of the model. A thin disk-shaped diffusion flame (modeled as an ionized layer) was stabilized in quasi onedimensional electric fields. Nozzles with a $10-\mathrm{mm}$ diameter were spaced $10 \mathrm{~mm}$ apart. At the 
exit of each nozzle, we placed a thin, perforated disk with a hole density of $79 / \mathrm{cm}^{2}$ and an 800 $\mu \mathrm{m}$ hole diameter to establish a quasi one-dimensional electric field by connection with a power supply [16].

To form diffusion flames between the two nozzles (also the electrodes), nitrogen-diluted fuel and oxygen streams were injected through the upper and lower nozzles, respectively. By changing the initial mole fractions of fuel and/or oxygen at the nozzle exits, the position of the diffusion flame relative to the lower nozzle could be adjusted keeping the same flame temperature [33]. Methane $\left(\mathrm{CH}_{4}\right)$, ethane $\left(\mathrm{C}_{2} \mathrm{H}_{6}\right)$, and propane $\left(\mathrm{C}_{3} \mathrm{H}_{8}\right)$ were tested, and detailed experimental conditions are listed in Table 1.

Overall mixture compositions in the flame zones due to the diffusion of the reactant were designed to have a similar adiabatic flame temperature of $2250 \mathrm{~K}$, which were calculated using the OPPDIF code of CHEMKIN-PRO package [34] with GRI 3.0 mechanism [35]; designed mixture compositions at the flame zones were $\mathrm{CH}_{4} / \mathrm{O}_{2} / \mathrm{N}_{2}=1 / 2 / 5.3, \mathrm{C}_{2} \mathrm{H}_{6} / \mathrm{O}_{2} / \mathrm{N}_{2}=1 / 3.5 / 8.7$, and $\mathrm{C}_{3} \mathrm{H}_{8} / \mathrm{O}_{2} / \mathrm{N}_{2}=1 / 5 / 11.7$, regardless of flame locations. Subsequently, the generation of ions in a unit volume of the flame could be maintained similarly for various flame locations, however, it might be different by fuel due to fuel dependent ion chemistry.

For a methane flame as an example, we stabilized the flame near the center when the mole fractions of nitrogen-diluted methane $\left(X_{\mathrm{CH} 4}\right)$ and oxygen $\left(X_{\mathrm{O} 2}\right)$ were set at 0.222 and 0.527 , respectively; both streams had a mean jet velocity of $20 \mathrm{~cm} / \mathrm{s}$ (Fig. 2a). However, to achieve an appreciable contrast in flame location, we adjusted the mean jet velocities to $30 \mathrm{~cm} / \mathrm{s}$ and 10 $\mathrm{cm} / \mathrm{s}$ with $X_{\mathrm{CH} 4}=0.348$ and $X_{\mathrm{O} 2}=0.369$ respectively, which moved the flame closer to the oxidizer nozzle (Fig. 2b).

We assumed that the location of generated ions would reasonably coincide with the flame front, typically indicated by a blue layer caused by the chemiluminescence of $\mathrm{CH}$ radicals. Thus, the brightest axial locations of the blue flames were considered to coincide with the locations of the thin, ionized layers, validating the model by experimentation.

Coaxial nitrogen-sheath gas surrounding the jets were supplied to minimize outside disturbance and to suppress a parasitic secondary diffusion flame with the ambient air. The mean flow velocity of the nitrogen sheath was matched with that of the corresponding central jet to suppress unnecessary effects of shear layer between the central jet and the sheath flow. The flow rates of gases were controlled with mass flow controllers. Water-cooled nozzles maintained the fuel and oxidizer at a consistent temperature. 
A power supply (Trek, 10/10B-HS) and a function generator (NF, WF1973) applied a DC electric potential to the lower nozzle, while the upper nozzle was grounded. An oscilloscope (Tektronics, DPO 2024) was used to monitor voltage and current. A 1000:1 voltage probe (Tektronics, P6015A) measured applied voltages, and an additional resistor of $100 \mathrm{k} \Omega$ was placed in series between the upper nozzle and the ground to measure the current. The voltage across the resistor was converted into current, which was corrected with respect to an internal resistance $(1 \mathrm{M} \Omega)$ of a voltage probe (Agilent, 10070C).

\section{Results and Discussion}

The current behaved asymmetrically [10, 26-29] when the DC was applied to the flame at different polarities. Potentially, the asymmetry was caused by a distortion to the local electric field because the ionized layer was skewed towards one electrode, and/or because of three-orders of-magnitude difference in mobility between electrons and ions.

\subsection{The ionized layer at the center of a gap}

First, we analyzed the behavior of an electric current computationally when a thin, ionized layer was located centrally between the electrodes $(H / L=0.5)$. Two extreme cases $-\alpha=0$ (all negative charges were carried by negative ions) and $\alpha=1$ (all negative charges were carried by electrons) - were examined to test the effect of different mobility. For both positive and negative ions, mobility was adopted at $2.9 \times 10^{-4} \mathrm{~m}^{2} / \mathrm{s}-\mathrm{V}[27,30]$, while electron mobility was set at 0.4

$\mathrm{m}^{2} / \mathrm{s}-\mathrm{V}$ [36]. The width parameter $a$ of the ion generation profile $g$ was set at $1 \mathrm{~mm}$ to approximate typical flame thickness. Ionization rate coefficient $k_{i}$ was selected as $1 \times 10^{20}$ ions $/ \mathrm{m}^{3}$-s [32] and recombination rate coefficient $k_{r}$ was $2.4 \times 10^{-13} \mathrm{~m}^{3} /$ ions-s [32].

Figure 3 shows the current density plotted against the mean field intensity defined as $V_{a} / L$. The simulated result captured the typical ion current characteristics in the sub-saturated regime as having a near-quadratic increase of trend for low-field intensity (best-fit quadratic curves are marked by dotted lines) and predicted a saturated regime for high-field intensity. Because a saturated current depends only on the rate of ion generation, the saturation current is independent of the type of negative charge carrier $(\alpha)$. The rate of increase in current density is much faster with a field intensity of $\alpha=1$ than with a field intensity of $\alpha=0$. 
A difference in the mobility of negatively charged particles (between negative ions and electrons) could cause a change in the distribution of space charge between the source of the charge and the anode. Consequently, locally modified field intensity due to space charge redistribution could provoke charged particles to have different dynamic responses. In this regard, the local field intensity $E$ is plotted against $x / L$ for $V_{a}= \pm 300 \mathrm{~V}$ to confirm its local modification. As a result, local field intensities with $\alpha=1$ are approximately twice those with $\alpha=0$, and subsequently, the aforementioned accelerated rate of current density increased at $\alpha=1$ compared to $\alpha=0$. However, the location of the ionized layer mirrored the profiles of local field intensity regardless of the $\alpha$ value for different polarities of an applied DC. This can be attributed to the fact that the ionized layer is located centrally between two electrodes, such that characteristics of the asymmetrical current cannot be observed for different polarities of an applied DC.

\subsection{Skewed ionized layer location}

Current density behavior was further analyzed computationally when the ionized layer was positioned away from the center, toward either one of the electrodes. Figure 5 shows the result of current density for $H / L=0.25$ and 0.75 with $\alpha=0$ and 1 . The profiles of current density were symmetric with polarity when ions carried all negative charges $(\alpha=0)$.

Alternatively, when electrons carried all negative charges $(\alpha=1)$, current density behavior was asymmetric with respect to the polarity for $H / L=0.25$. For $H / L=0.75$, a conjugate condition of $H / L=0.25$ with respect to the central plane of symmetry, a steeper increase in the current was found when the energized electrode, which is farther from the ionized layer, applied positive potentials to the ionized layer.

Figure 5 illustrates the plane of symmetry in the electric current behavior for $H / L=0.25$ and 0.75 . Thus, when the ionized layer is skewed, the lower electric potential of an electrode closer to the ionized layer than that of one farther from the electrode induced a more rapid increase in the electric current.

Figure 6a demonstrates the results of testing the effect of $\alpha$ at several values with $H / L=$ 0.25. For $V_{a} / L>0$, the dependence of $\alpha$ on the current behavior was small, while for $V_{a} / L<0$ a drastic change in behavior was observed. As the negative potential applied to the energized electrode increased for a specified $\alpha$, (e.g., $\alpha=0.4$ ), initially the current rapidly increased as we observed for $\alpha=1$, but then only slowly increased, as we observed for $\alpha=0$. 
The bifurcation point where current behavior begins to branch out from the trend of $\alpha=1$ is marked in Fig. 6a with an open black dot. The normalized critical current for each tested $\alpha$ at its corresponding bifurcation point with a saturated current is reasonably matched with the proportion of electrons out of negative charge carriers (i.e., $\alpha$ ).

In Fig. 6b, we plotted the respective contributions of ions and electrons to current density for $\alpha=0.4$. The result shows that as $V_{a} / L$ decreases in the negative voltage range, the current density follows the behavior for $\alpha=1$, until the contribution by electrons is saturated. An additional increase in the current with increased negative $V_{a}$ beyond the critical point is attributed to negative ions. This indicates that the faster moving electrons migrate from the ionized layer first, followed by the slower moving negative ions. Note that in the positive potential region, no significant variation was found. However, the detailed bifurcation characteristics are the same as the cases in the negative potential region.

To further elucidate the effect of the difference in the mobility between ions and electrons and the skewed flame position on the development of an asymmetric current, the local electric potential $V$ and the field intensity $E$ were plotted against $x / L$ (Fig. 7). We compared $V_{a}=300 \mathrm{~V}$ and $-300 \mathrm{~V}$ when the ionized layer was located at $H / L=0.25$. To better explain this result, we introduced a thickness of the zone between the ion source and the anode $(\delta$-) (Fig. 7a), ideally filled with negative charges with a bias voltage. Likewise, $\delta_{+}$indicates the positively charged thickness between the source and the cathode. The subscript NP indicates that a negative potential was applied to the energized electrode, while PP represents a positive potential was applied to the energized electrode.

When $\alpha=0$, since the mobility of the positively charged (positive ion) and the negatively charged (negative ion) carriers are the same, the moving speed of each charge carrier from the source toward the corresponding electrodes is the same for the same field intensity. Thus, the number density of the negative ion in the zone between the source and the anode is the same as that of the positive ion in the region between the source and the cathode. Consequently, the absolute value of the local potential shows a point-wise symmetric behavior centered at the position of the ionized layer $(x=2.5 \mathrm{~mm})$, regardless of polarity. This is confirmed by the symmetric local field intensities shown in Fig. 7b, and evidences that at $\alpha=0$, the rates of increase in the current are the same irrespective of the polarity (Fig. 5).

On the other hand, when $\alpha=1$, due to faster moving electrons, the number density of electrons in the region between the source and the anode is much smaller than that of the positive 
ion in the region between the source and the cathode. This explains the resultantly less significant potential drop, due to space charges, in the zone between the source and the anode $\left(\delta_{\text {- }}\right)$ than between the source and the cathode $\left(\delta_{+}\right)$, where, as a counter effect, potential drop $\left(\sim V_{a}\right)$ compensation is necessary. For this reason, when a negative external potential is applied to the energized electrode, which is closer to the ionized layer than the other, the field intensity $\left(\sim V_{a} / \delta_{+, \mathrm{NP}}\right)$ could be enhanced, which is greater than $V_{a} / \boldsymbol{\delta}_{+, \mathrm{PP}}$ with a positive external potential. Figure $7 \mathrm{~b}$ clearly demonstrates this type of heightened local field intensity due to faster moving electrons. This disparity is what creates the asymmetric behavior of the rate of increase in the current reported in previous studies [10, 26-29] and obtained from our model (Fig. 5) ( $\alpha=1)$.

From Fig. 3, we observed that the symmetrical increase in electric current was predicted even with $\alpha=1$ when the ionized layer locates at the center of the gap between two electrodes $(H / L=0.5, H=5 \mathrm{~mm})$. Furthermore, the centered ionized layer dictates that the thicknesses between the ion source and the cathode are the same regardless of the polarity of the DC as $\delta_{+, \mathrm{NP}}$ $=\delta_{+, \mathrm{PP}}=5 \mathrm{~mm}$. Thus, the local field intensity along the gap has to be in plane symmetry with respect to the center of the gap (Fig. 4). Therefore, no asymmetric current behavior was found with a centered ionized layer.

Based on these results, we conclude that differences in mobility play a key role towards increasing the sensitivity of an electric current to an external voltage, particularly when the relative electric potential of a closer electrode to the ionized layer is lower than that of the other electrode. However, it should be noted that the migrated electrons may attach to neutral species, such as oxygen, to form negative ions. A rate of the electron attachment may depend on the electronegativities of neutral species, a gas density, and a traveling distance of the electrons [37]. Such electron attachment could not be considered in the present model.

\subsection{Model validation with counterflow diffusion flames}

To test the model empirically, experiments were performed with $H / L \sim 0.5$, simulating a centered ionized layer, and with $H / L=0.24$, representing an ionized layer that is skewed from the energized electrode (Table 1). A visible flame width was monitored at each applied voltage, and the projected area of the flame to the electrode was used to convert measured electric current into current density.

When the flames were located near the center, our model predicted the current behavior qualitatively well at $\alpha=1$, as shown in Fig. 8a, demonstrating a near symmetric current increase 
with polarity. Although the experimental data show little deviation with a negative DC, there is no significant difference in the current behaviors among tested fuels as a whole. It should be noted that there should be small effects due to fuel dependent ion-chemistry, because it has been well understood that the ion production reasonably scales with total carbon influx [38]. The fuels were used at different levels of dilution showing similar carbon flux for each fuel, which could mitigate any fuel effect on the ion production.

However, when the flames were positioned closer to the energized electrode $(H / L=0.24)$, these flames resulted in different responses of current even though they all demonstrated asymmetric trends with change in polarity (Fig. 8b). Overall, our model qualitatively well predicted the experimental asymmetric current behaviors better at $\alpha=1$ than at $\alpha=0$. No aforementioned bifurcation in current behavior was observed with a negative DC, implying that most negative charges are carried by electrons in flames or that the amount of flame-generated negative ions are much smaller than that of positive ions (Fig. 6).

The discrepancy between the model and experimental results in Fig. 8 for the current behavior may mainly be attributed to the effect of an electric field on diffusion flames, because it can also significantly alter the structure of a flame [16]. Further detailed flame simulation coupled with Poisson's equation including ion-chemistry is needed in the near future.

\subsection{Sensitivity Analysis}

In the above sections with the simple one-dimensional modeling, we concluded that the difference in the mobility among charge carriers and the relative location of the ion source in between electrodes are the key factors in asymmetric electric current responses with DC. To support our conclusion, we conducted a parametric study, which shows how the trend of predicted voltage-current (V-I) behavior is sensitive to a selected parameter.

To explore the sensitivity of the electric current due to variation in each parameter, we nondimensionalized the governing equations referring to [20] using $n_{0}=\left(k_{i} / k_{r}\right)^{1 / 2}, L_{0}=L$, $t_{0}=\varepsilon / e K_{+} n_{0}, V_{0}=e n_{0} L^{2} / \varepsilon$ as the characteristic scales for number density, length, time, and voltage, respectively. Then, non-dimensionalized variables were presented as: $\tilde{x}=x / L_{0}$, $\tilde{t}=t / t_{0}, \tilde{n}=n / n_{0}$, and $\tilde{V}=V / V_{0}$.

Substituting above non-dimensional variables into the dimensional governing equations, associated non-dimensional forms can be expressed as: 
Positive ions: $\quad \frac{\partial \tilde{n}_{+}}{\partial \tilde{t}}+\frac{\partial}{\partial \tilde{x}}\left[-\gamma_{+}^{2} \frac{\partial \tilde{n}_{+}}{\partial \tilde{x}}-\tilde{n}_{+} \frac{\partial \tilde{V}}{\partial \tilde{x}}\right]=\kappa^{2}\left[g-\tilde{n}_{+}\left(\tilde{n}_{e}+\tilde{n}_{-}\right)\right]$

Negative ions: $\quad \frac{\partial \tilde{n}_{-}}{\partial \tilde{t}}+\frac{\partial}{\partial \tilde{x}}\left[-\gamma_{-}^{2} \frac{\partial \tilde{n}_{-}}{\partial \tilde{x}}+R_{-} \tilde{n}_{-} \frac{\partial \tilde{V}}{\partial \tilde{x}}\right]=\kappa^{2}\left[(1-\alpha) g-\tilde{n}_{+} \tilde{n}_{-}\right]$,

Electrons:

$$
\frac{\partial \tilde{n}_{e}}{\partial \tilde{t}}+\frac{\partial}{\partial \tilde{x}}\left[-\gamma_{e}^{2} \frac{\partial \tilde{n}_{e}}{\partial \tilde{x}}+R_{e} \tilde{n}_{e} \frac{\partial \tilde{V}}{\partial \tilde{x}}\right]=\kappa^{2}\left(\alpha g-\tilde{n}_{+} \tilde{n}_{e}\right)
$$

Electric potential: $\quad \frac{\partial^{2} \tilde{V}}{\partial \tilde{x}^{2}}=-\left(\tilde{n}_{+}-\tilde{n}_{-}-\tilde{n}_{e}\right)$

Spatial profile of ionization: $g=\exp \left[-\frac{\pi(\tilde{x}-\varphi)^{2}}{\eta^{2}}\right]$

where $\gamma_{k}^{2}=D_{k} t_{0} / L_{0}^{2}=D_{k} \varepsilon / L_{0}^{2} e K_{+} n_{0}$ (dimensionless diffusivity), $R_{k}=K_{k} / K_{+}$(mobility ratio), $k=+,-$, and $e$ for $\gamma_{k}$, and - and $e$ for $R_{k}, \varphi=H / L_{0}$ (scaled ion source location), $\eta=a / L_{0}$ (scaled ion source thickness), and $\kappa^{2}=k_{i} t_{0} / n_{0}=\varepsilon k_{r} / e K_{+}$(generalized dimensionless ion rate coefficient).

The non-dimensional boundary conditions are,

$$
\tilde{V}(\tilde{x}=0, \tilde{t})=V_{a} / V_{0}, \tilde{V}(\tilde{x}=1, \tilde{t})=0, \tilde{n}_{k}(\tilde{x}=0, \tilde{t})=0, \tilde{n}_{k}(\tilde{x}=1, \tilde{t})=0,
$$

where $k=+,-$, and $e$.

Figure 9 shows the result of the sensitivity test with $\alpha=1$, emphasizing significant dependence of the electric current response on the scaled ion source location $(\varphi)$ in the subsaturated regime (Fig. 9f). The baseline case in each sub-figure represents the dimensional solution in Fig. 6a with $\alpha=1$. The dimensionless diffusivities $\left(\gamma_{+}^{2}\right.$ and $\left.\gamma_{e}^{2}\right)$ do not change the overall trend of the current with $\pm 50 \%$ perturbation of each parameter from the baseline case (Fig. 9a and b). This indicates that the mass diffusion is secondary compared to the transportation of charged species due to external electric fields. Negligible influence of the perturbation is found with the mobility ratio $\left(R_{e}\right)$ (Fig. 9c). Recalling that the mobility of the electron is three orders of magnitude higher than that of positive ions, $50 \%$ reduction of $R_{e}$ still 
means two orders of difference in the mobilities. Thus, a factor of variation in the positive ion mobility, which might be varied by different species and temperature, cannot affect the overall asymmetric trend of the baseline case.

Perturbation of the generalized dimensionless ion rate coefficient $\left(\kappa^{2}\right)$ by $50 \%$ resulted in significant modification of the saturation current and slight change in the rate of current increase (Fig. 9d). Specifically, when $\kappa^{2}$ increases, the saturation current increases demonstrating rapid increase in the sub-saturated regime compared to the baseline case. However, an overall asymmetric trend of the current is not affected. Similar effect on the saturation current can also be obtained by varying the scaled ion source thickness $(\eta)$ (Fig. 9e). It is natural to expect that a large amount of generated ions with a thicker ion layer lead to increased level of the saturated current. The adjustment in the rate of increase in the sub-saturated regime was not found unlike the case with $\kappa^{2}$.

Finally, the scaled ion source location $(\varphi)$ can affect the symmetry of the V-I curve noticeably (Fig. 9f). The rotation of the V-I curve is easily identified showing a transition from a symmetric to an asymmetric trend when $\varphi$ becomes smaller. This analysis is consistently supporting our previous conclusion that the relative location of the ion source is one of the two important reasons to show an asymmetric V-I trend.

Figure 10 shows dimensional sensitivities due to dimensional parameters, which effectively demonstrates only the mobility difference between positive and negative charge carriers can lead to the asymmetric V-I trend. Again, the baseline case in each sub-figure represents the dimensional solution in Fig. 6a with $\alpha=1$.

Increase in the rate of ion generation due to increased $k_{i}$ resulted in a higher saturation current (Fig. 10a) because $k_{i}$ directly relates to the total amount of generated ions. However, the rate of ion recombination is not important to the V-I trend (Fig. 10b), because the separation of charge carriers toward opposite directions significantly reduces a recombination possibility regardless of $k_{r}$. The mobility of the positive ions affects the rate of increase in the current in the sub-saturated regime (Fig. 10c), but it does not change the overall asymmetric V-I trend with $\alpha=$ 1.

Finally we found that the main factor to make the V-I trend be asymmetric is the difference in mobilities between positive and negative charge carriers (Fig. 10d). Assuming all negative charges was carried by single species, the sensitivity of the V-I trend due to the mobility of the negative charge carrier can be found. In Fig. $10 \mathrm{~d}, K_{-} / K_{+}=1$ implies the case with $\alpha=0$ and the 
baseline indicates the case with $\alpha=1$. For an arbitrary mobility of the negative charge carrier, represented by $K_{-} / K_{+}=10$ and 100 , the sensitivity test shows a limiting behavior as the ratio increases. As a result, we found that the increase in the mobility of negative charge carrier is the other key factor to the asymmetric V-I trend, which consistently supports our conclusion in the section 4.2.

\section{Conclusions}

Our model highlights the behavior of an electric current with an external electric potential applied to a thin, ionized layer. Because electrons move faster than positive ions, a major potential drop occurs in the region between an ion source and a lower potential side (cathode). Therefore, when the ionized layer becomes closer to the cathode, effective electric field intensity at the region between the ion source and the cathode becomes stronger. For this reason, an asymmetric current increase occurs at different polarities, showing a more rapid increase in the current with a negative external potential applied to the electrode that is closer to the ion layer. We qualitatively validated the model here by comparing it with experimental results using counterflow diffusion flames. A sensitivity analysis with selected dimensional and nondimensional parameters also supported the importance of the mobility difference and the relative location of the ion layer on the asymmetric V-I behavior. Meanwhile, the mobility of positive ions affected the increasing rate of the current in the sub-saturated regime, when electrons carried all negative charges.

Finally, we could reasonably explain the qualitative responses of the electric current to the DC by modeling a flame as an ionized layer, even without considering detailed ion chemistry. However, to substantiate related flow modifications due to ionic wind and consequent flame responses, comprehensive simulation efforts are necessary including ionic wind effect as well as relevant ion chemistry in the future.

\section{Acknowledgment}

The research reported in this publication was supported by Competitive Research Funding from King Abdullah University of Science and Technology (KAUST).

\section{References}


[1] Y. Xiong, M.S. Cha, S.H. Chung, Proc. Combust. Inst. 35 (2015) 3513-3520.

[2] E.N. Volkov, V.N. Kornilov, L.P.H. De Goey, Proc. Combust. Inst. 34 (2013) 955-962.

[3] M.K. Kim, S.H. Chung, H.H. Kim, Combust. Flame 159 (2012) 1151-1159.

[4] M.S. Cha, Y. Lee, IEEE Trans. Plasma Sci. 40 (2012) 3131-3138.

[5] M.K. Kim, S.H. Chung, H.H. Kim, Proc. Combust. Inst. 33 (2011) 1137-1144.

[6] G.K. Ryu, Y.K. Kim, M.K. Kim, S.H. Won, S.H. Chung, Combust. Flame 157 (2010) 25-32.

[7] J.D.B.J. Van Den Boom, A.A. Konnov, A.M.H.H. Verhasselt, V.N. Kornilov, L.P.H. De Goey, H. Nijmeijer, Proc. Combust. Inst. 32 (2009) 1237-1244.

[8] S.H. Won, S.K. Ryu, M.K. Kim, M.S. Cha, S.H. Chung, Combust. Flame 152 (2008) 496506.

[9] M. Sánchez-Sanz , D.C. Murphy, C. Fernandez-Pello, Proc. Combust. Inst. 35 (2015) 34633470 .

[10] S.D. Marcum, B.N. Ganguly, Combust. Flame 143 (2005) 27-36.

[11] H. Jaggers, A. Von Engel, Combust. Flame 16 (1971) 275-285.

[12] F. Altendorfner, A. Sakhrieh, F. Beyrau, A. Leipertz, F. Dinkelacker, G. Lins, T. Hammer, D. Branston, Proc. Euro. Combust. Meeting (2007) Greece.

[13] M. Saito, T. Arai, M. Arai, Combust. Flame 119 (1999) 356-366.

[14] M. Saito, M. Sato, K. Sawada, J. Electrostat. 39 (1997) 305-311.

[15] A. Vatazhin, V. Likhter, V. Sepp, V. Shul'Gin, Fluid Dynamics 30 (1995) 166-174.

[16] D.G. Park, B.C. Choi, M.S. Cha, S.H. Chung, Combust. Sci. Tech. 186 (2014) 644-656.

[17] A.P. Chattock, Philos. Mag. 48 (1899) 401-420.

[18] F.B. Carleton, F.J. Weinberg, Nature 330 (1987) 635-636.

[19] M. Belhi, P. Domingo, P. Vervisch, Combust. Flame 157 (2010) 2286-2297.

[20] A.M. Drews, L. Cademartiri, M.L. Chemama, M.P. Brenner, G.M. Whitesides, K.J. Bishop, Phys. Rev. E 86 (2012) 036314.

[21] S. Karnani, D. R. Rankin, Combust. Flame 162 (2015) 2865-2872

[22] A. Sakhrieh, G. Lins, F. Dinkelacker, T Hammer, A. Leipertz, D.W. Branston, Combust. Flame 143 (2005) 313-322

[23] J. Lawton, F.J. Weinberg, Electrical aspects of combustion, Clarendon Press, Oxford, 1969.

[24] F. Borgatelli, D. Dunn-Rankin, Combust. Flame 159 (2012) 210-220.

[25] K. Yamashita, S. Karnani, D. Dunn-Rankin, Combust. Flame 156 (2009) 1227-1233.

[26] M.J. Papac, Electrical aspects of gaseous fuel flames for microgravity combustion and combustion control, University of California Irvine, USA, 2005. 
[27] J. Vinogradov, E. Sher, I. Rutkevich, M. Mond, Combust. Flame 127 (2001) 2041-2050.

[28] D. Bradley, F.J. Weinberg, Advance Combustion Methods, Academic Press, London, 1986.

[29] H. Calcote, R. Pease, Ind. Eng. Chem 43 (1951) 2726.

[30] M.J. Papac, D. Dunn-Rankin, Combust. Theor. Model. 12 (2008) 23-44.

[31] A. Hospital, P. Roth, Proc. Combust. Inst. 23 (1991) 1573-1579.

[32] A.B. Fialkov, Prog. Energy Combust. Sci. 23 (1997) 399-528.

[33] M.S. Cha, P.D. Ronney, Combust. Flame 146 (2006) 312-328.

[34] CHEMKIN-PRO Package, Reaction Design, 6440 Lusk Boulevard, Suite D-205 San Diego, CA 92121. <http://www.reactiondesign.com>.

[35] G.P. Smith, D.M. Golden, M. Frenklach, N.W. Moriarty, B. Eiteneer, M. Goldenberg, C.T. Bowman, R.K. Hanson, S. Song, W.C. Gardiner Jr., V.V. Lissianski, Z. Qin, http://www.me.berkeley.edu/gri mech/.

[36] F. Bisetti, M. El Morsli, Combust. Flame 159 (2012) 3518-3521.

[37] F.J. Weinberg, D.R. Rankin, F.B. Carleton, S. Karnani, C. Markides, M. Zhai, Proc. Combust. Inst. 34 (2013) 3295-3301

[38] D. Boothman, J. Lawton, S.J. Melinek, F. Weinberg, Proc. Combust. Inst. 12 (1969) 969978 
Table 1. Experimental conditions validating the model.

\begin{tabular}{cccccccc}
\hline & \multirow{2}{*}{$\begin{array}{c}H \\
\text { Fuel }\end{array}$} & \multicolumn{3}{c}{ Upper Nozzle } & \multicolumn{3}{c}{ Lower Nozzle } \\
\cline { 3 - 8 } & {$[\mathrm{mm}]$} & $X_{\text {fuel }}$ & $X_{\mathrm{N} 2}$ & $\begin{array}{c}U \\
{[\mathrm{~cm} / \mathrm{s}]}\end{array}$ & $X_{\mathrm{O} 2}$ & $X_{\mathrm{N} 2}$ & $\begin{array}{c}U \\
{[\mathrm{~cm} / \mathrm{s}]}\end{array}$ \\
\hline $\mathrm{CH}_{4}$ & 4.9 & 0.222 & 0.778 & 20 & 0.527 & 0.473 & 20 \\
& 2.4 & 0.348 & 0.652 & 30 & 0.369 & 0.631 & 10 \\
\hline $\mathrm{C}_{2} \mathrm{H}_{6}$ & 5.1 & 0.147 & 0.853 & 20 & 0.548 & 0.452 & 20 \\
& 2.4 & 0.246 & 0.754 & 30 & 0.383 & 0.617 & 10 \\
\hline & 5.1 & 0.112 & 0.888 & 20 & 0.570 & 0.430 & 20 \\
$\mathrm{C}_{3} \mathrm{H}_{8}$ & 2.4 & 0.195 & 0.805 & 30 & 0.398 & 0.602 & 10 \\
\hline
\end{tabular}

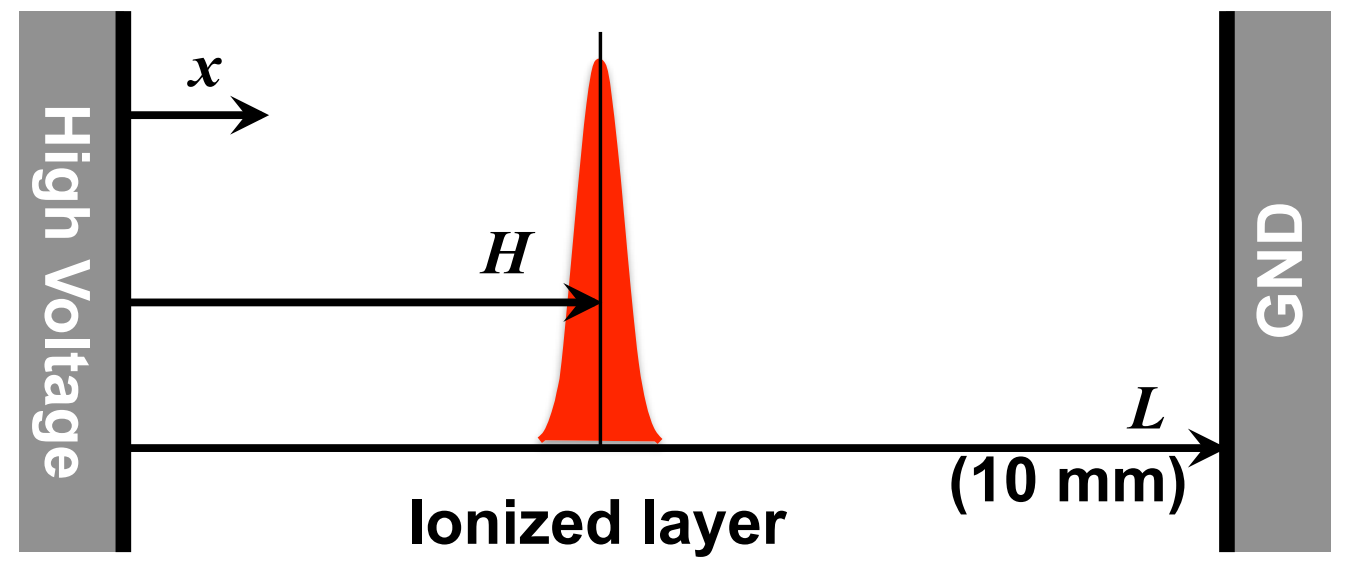

Figure 1. Schematic of a model ionized layer in a one-dimensional electric field. 


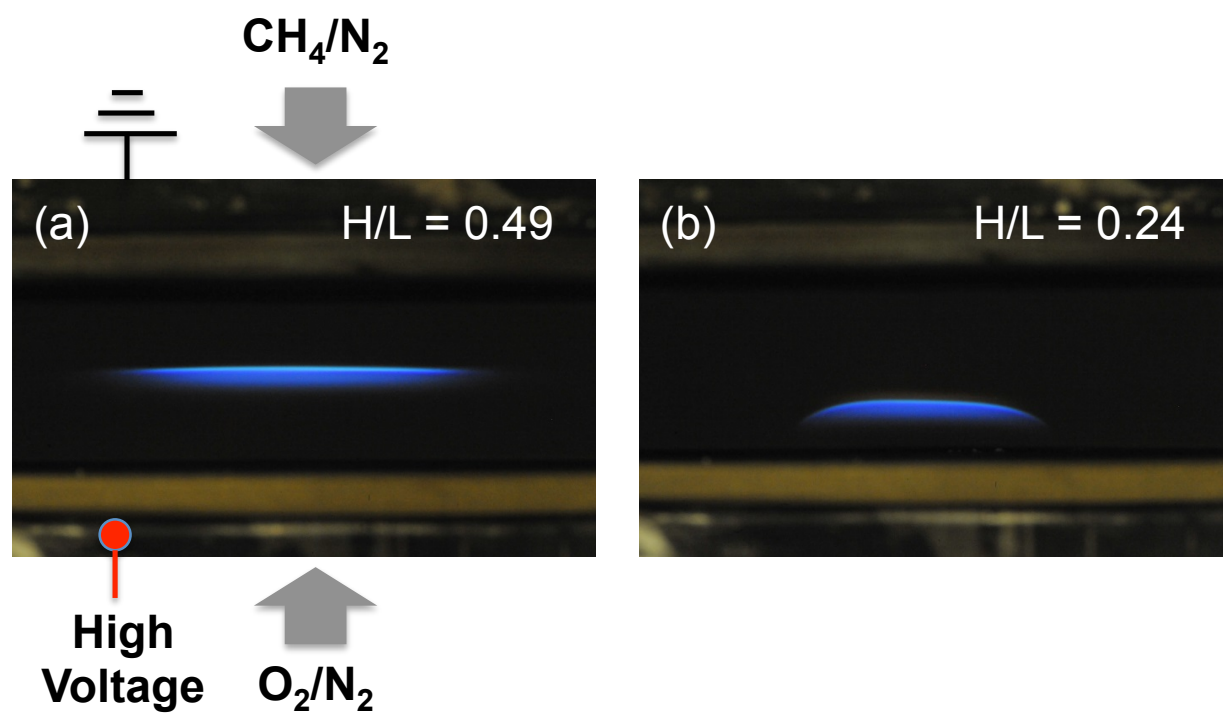

Figure 2. Non-premixed flames in a counterflow burner validating the model for a thin, ionized layer subjected to a one-dimensional electric field by applying an external voltage to the lower nozzle. Detailed experimental conditions are presented in Table 1.

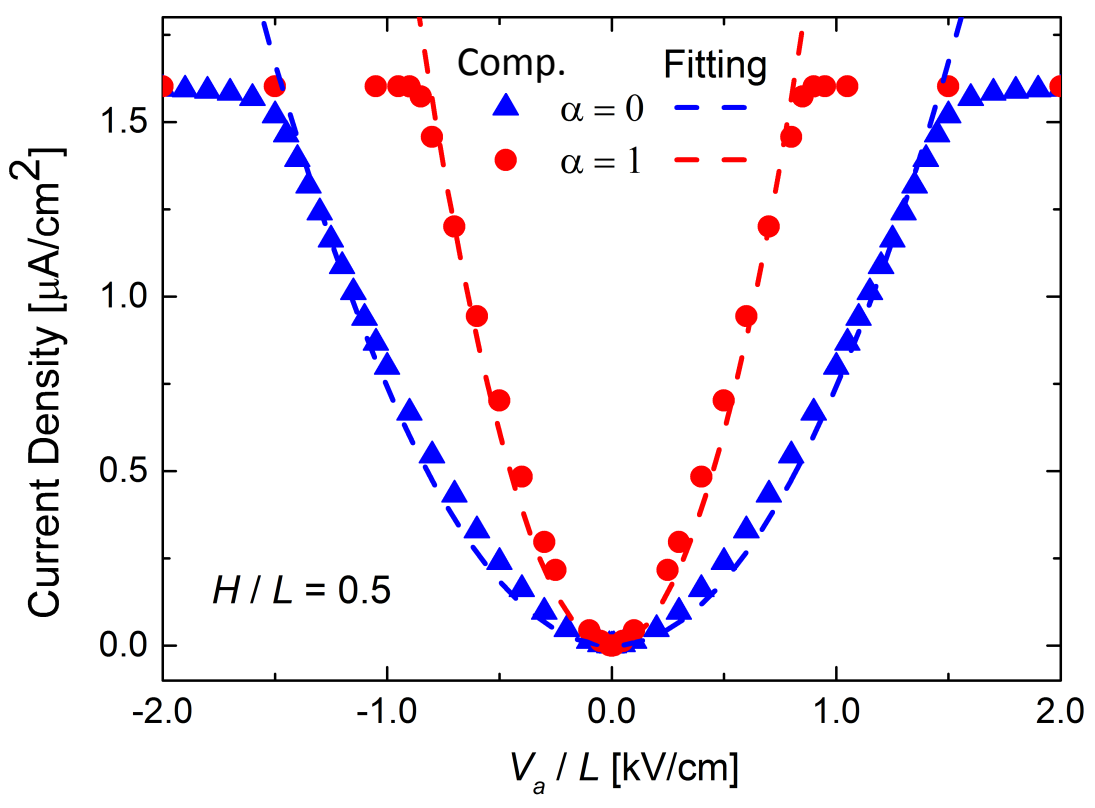

Figure 3. Calculated current density against field intensity for $H / L=0.5$. Negative $V_{a} / L$ values indicate a negative applied voltage to the energized electrode. The dashed lines represent the line of best fit in a quadratic form. 


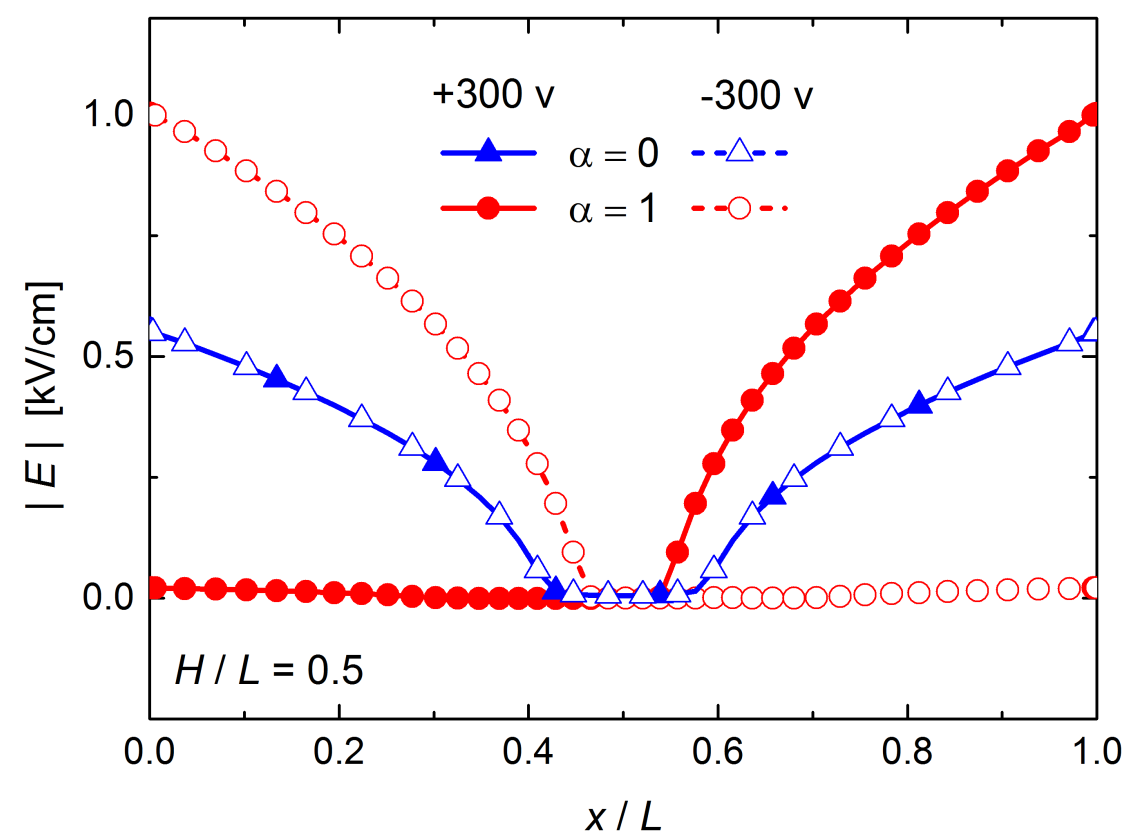

Figure 4. Calculated spatial profiles of local field intensity for $H / L=0.5$. Closed and open symbols indicate $V_{a}=300 \mathrm{~V}$ and $1-300 \mathrm{~V}$, respectively.

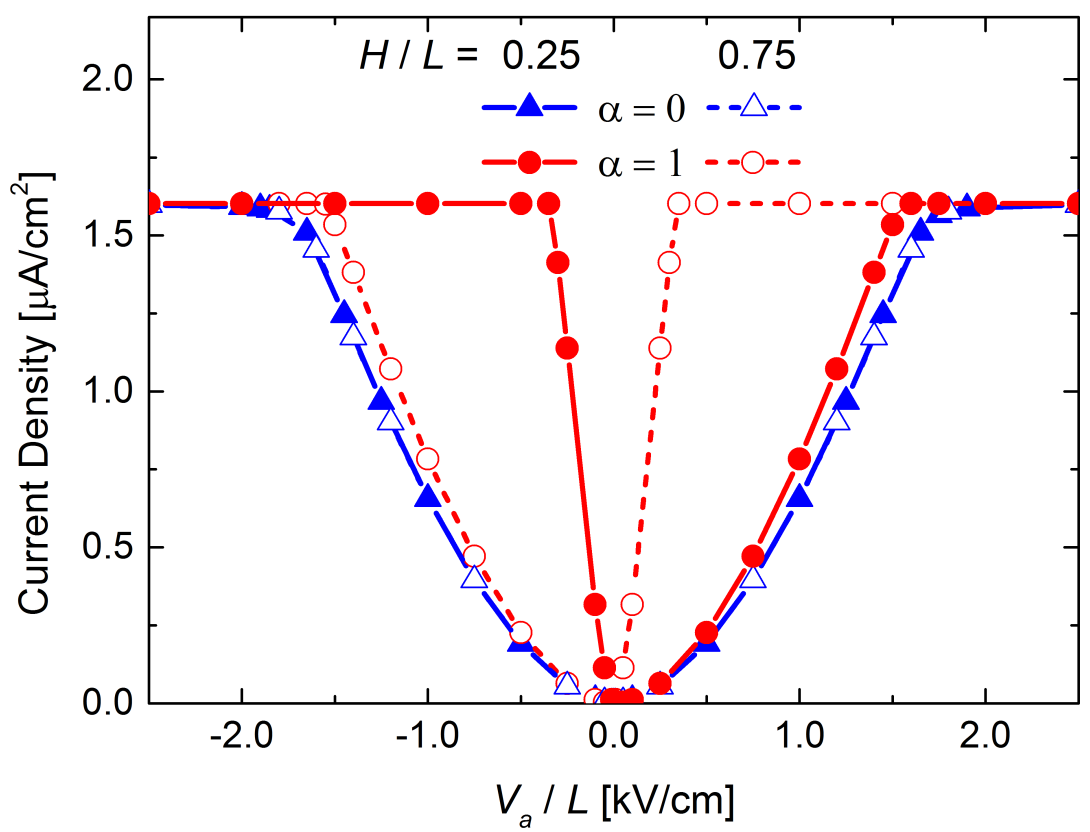

Figure 5. Calculated current density along with the field intensity for $H / L=0.25$ and 0.75 . Negative $V_{a} / L$ values indicate negative applied voltage to the energized electrode. 

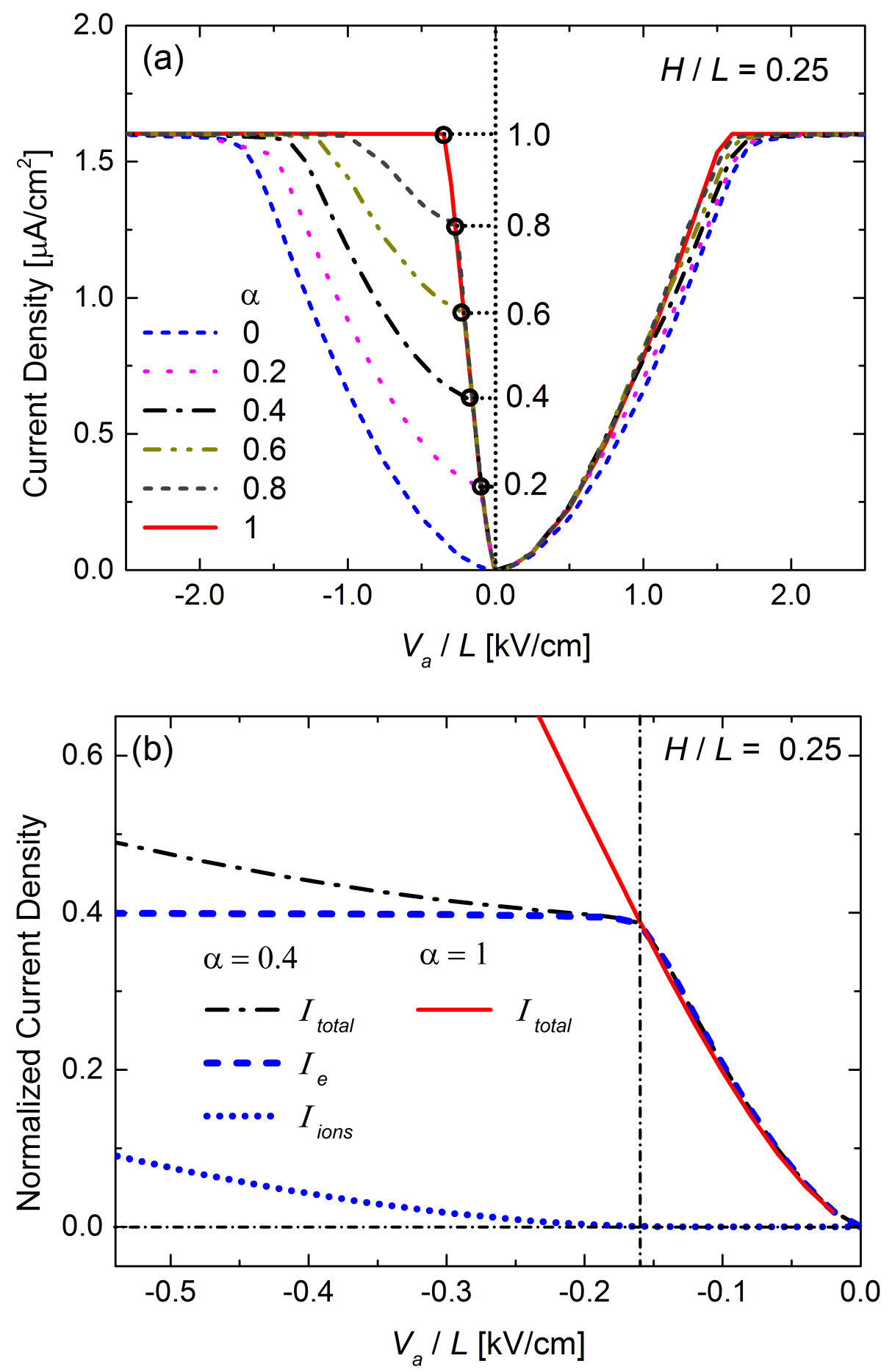

Figure 6. Calculated current density against field intensity for $H / L=0.25$ with various ratios of electrons to total negative charge carriers (a). $\alpha=1$ indicates electrons carry all negative charges, while $\alpha=0$ indicates all negative charges are carried by negative ions; A $\alpha=0.4$ evidences that faster moving electrons migrate earlier than negative ions (b). Negative $V_{a} / L$ values indicate negative applied voltage to the energized electrode. 

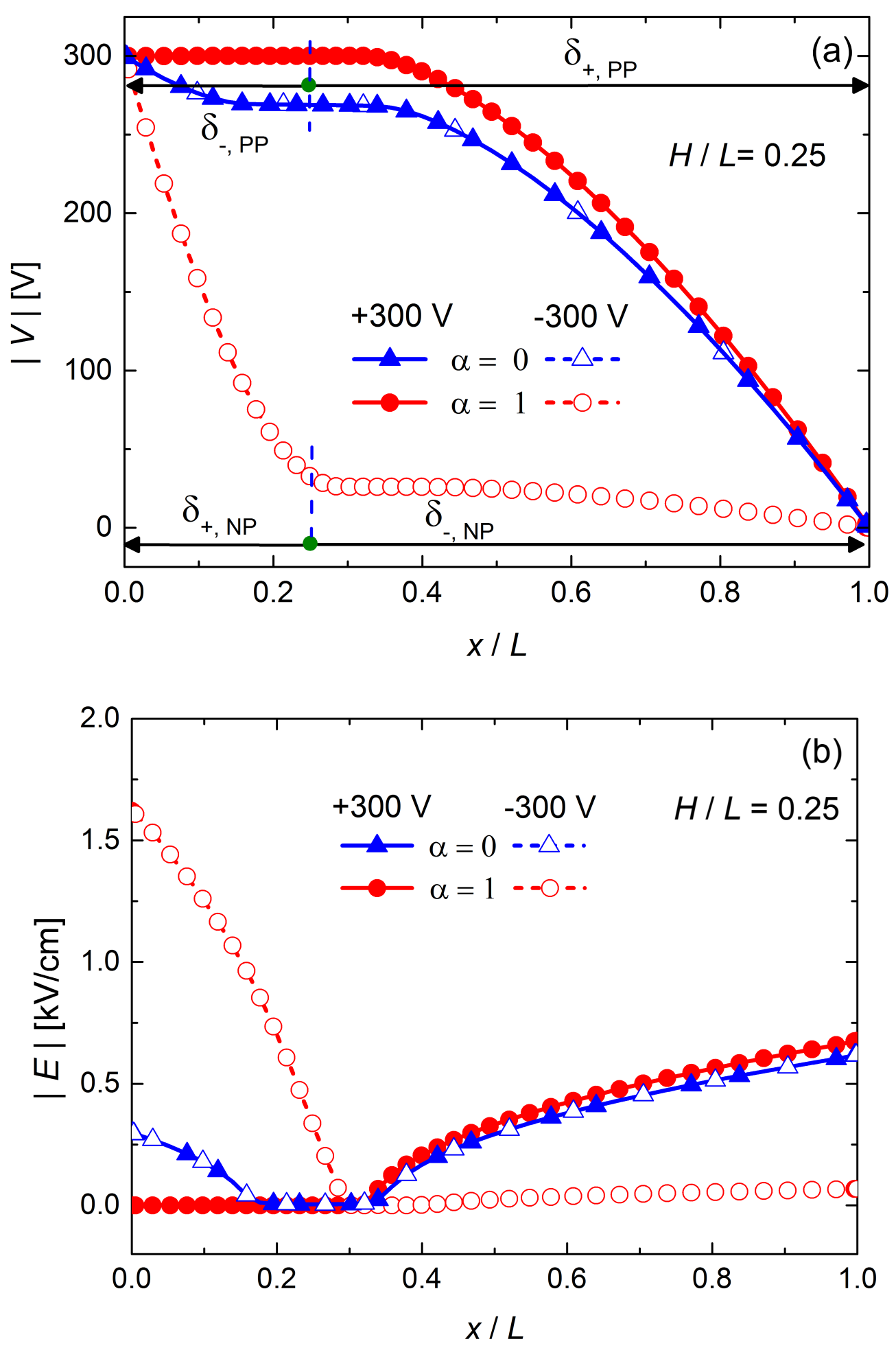

Figure 7. Calculated potential (a) and field intensity (b) with the location from the energized electrode for $H / L=0.25 . \alpha=1$ indicates electrons carry all negative charges, while $\alpha=0$ indicates all negative charges are carried by negative ions. $V_{a}=300 \mathrm{~V}$ and $-300 \mathrm{~V}$ are included for comparison. 

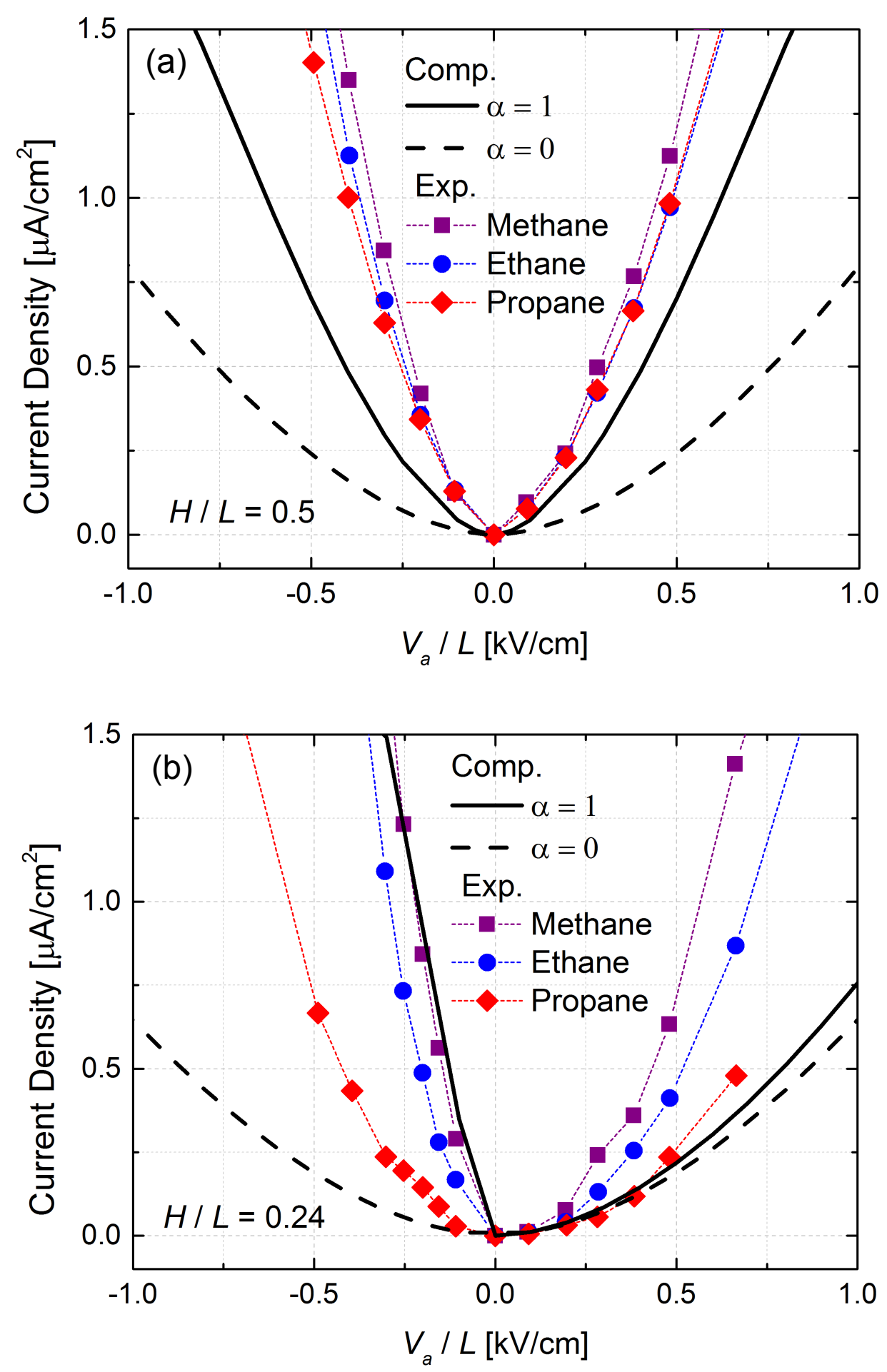

Figure 8. A comparison of model simulations with counterflow diffusion flames for $H / L=0.49$ (a) and 0.21 (b). $\alpha=1$ indicates electrons carry all negative charges, while $\alpha=0$ indicates all negative charges are carried by negative ions. 

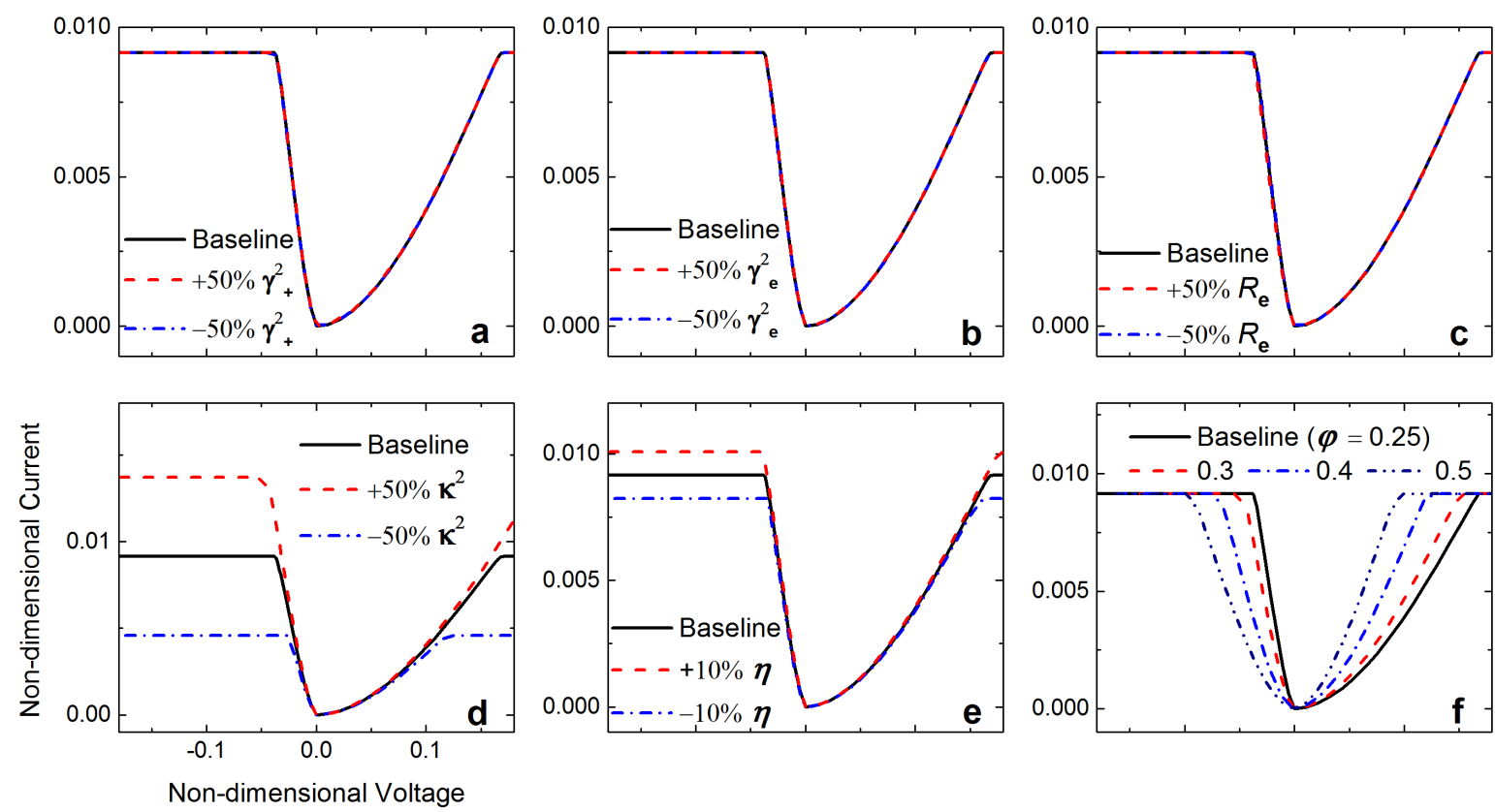

Figure 9. Effects of non-dimensional parameters on the non-dimensional V-I characteristics.
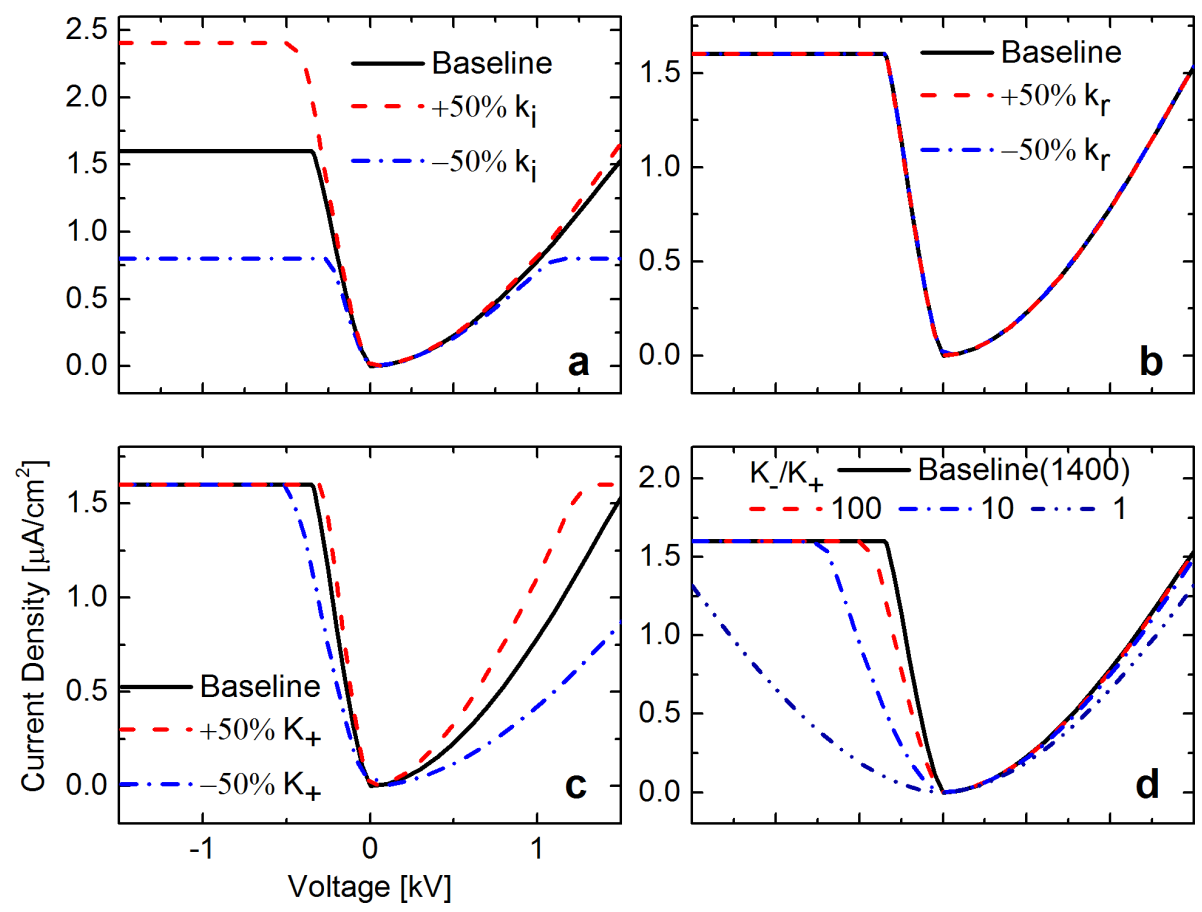

Figure 10. Effects of dimensional parameters on the V-I characteristics. 